Arq. Bras. Med. Vet. Zootec., v.69, n.4, p.883-888, 2017

\title{
Herniorrafia diafragmática videoassistida em gato: relato de caso
}

\author{
[Videoassisted diaphragmatic hernia repair in a cat: case report] \\ B. Copat ${ }^{1}$, B. Bertoletti ${ }^{2}$, R.O. Chaves ${ }^{3}$, J.P.S. Feranti ${ }^{2}$, G.Coradini ${ }^{2}$, \\ H.F. Hartmann ${ }^{2}$, L.F.D. Corrêa ${ }^{2}$, M.V. Brun ${ }^{2,4^{*}}$ \\ ${ }^{1}$ Médico veterinário residente - Universidade Luterana do Brasil - Ulbra - Canoas, RS \\ ${ }^{2}$ Universidade Federal de Santa Maria - UFSM - Santa Maria, RS \\ ${ }^{3}$ Universidade de Caxias do Sul - UCS - Caxias do Sul, RS \\ ${ }^{4}$ Bolsista de produtividade do $\mathrm{CNPq}$
}

\begin{abstract}
RESUMO
Relata-se um caso de um felino com sinais de dificuldade respiratória havia cerca de 30 dias e emagrecimento progressivo. Ao exame radiográfico torácico e de abdômen, ficou evidenciada perda de definição da linha diafragmática, sendo compatível com hérnia diafragmática. Na laparoscopia, foi observado grande defeito diafragmático, além de estruturas herniadas. Em razão de as vísceras abdominais direcionarem-se ao tórax, dificultando a oclusão do defeito com suturas intracorpóreas, optou-se pela realização de incisão paracostal, procedendo-se à herniorrafia por celiotomia reduzida. A videolaparoscopia permitiu localizar o local herniado e promover a redução dos órgãos envolvidos com mínimo trauma operatório. Logo, mostrou-se como eficaz ferramenta diagnóstica e auxiliar no tratamento de hérnias diafragmáticas crônicas de grande dimensão em gato.
\end{abstract}

Palavras-chave: felino, hérnia diafragmática, cirurgia minimamente invasiva

\begin{abstract}
This is a case report of a cat with signs of respiratory distress for about 30 days and progressive weight loss. The thoracic radiograph and abdomen examination evidencing loss of definition of the diaphragmatic line is compatible with diaphragmatic hernia. In laparoscopy, a large diaphragmatic defect and herniated structures were observed. Since abdominal viscera point towards the chest making occlusion of defective intracorporeal sutures difficult, paracostal incision proceeding to hernia repair by short celiotomy was chosen. Laparoscopy allows for location of herniated place and promotes the reduction of organs involved with minimal surgical trauma. Therefore, it was shown to be an effective diagnostic tool and an aid in the treatment of chronic diaphragmatic hernias of large size in a cat.
\end{abstract}

Keywords: feline, diaphragmatic hernia, minimally invasive surgery

\section{INTRODUÇÃO}

Hérnia diafragmática é a protrusão de órgãos abdominais para a cavidade torácica por meio de uma ruptura do diafragma. Pode ser congênita ou adquirida, sendo essa última a apresentação mais comum em cães e gatos. Entre as causas mais frequentes de hérnia diafragmática adquirida, incluem-se traumas por acidentes automobilísticos, quedas, chutes e brigas (Hage e Iwasaki, 2001; Schmiedt et al., 2003; Minihan et

Recebido em 2 de outubro de 2016

Aceito em 10 de outubro de 2016

*Autor para correspondência (corresponding author)

E-mail: mauriciovelosobrun@hotmail.com al., 2004; Gibson et al., 2005; Besalti et al., 2011).

Sinais clínicos de hérnia diafragmática incluem: dispneia, cianose, abafamento de sons cardiopulmonares a auscultação e choque, entretanto muitos pacientes podem apresentar apenas sinais inespecíficos, como anorexia, letargia e perda de peso (Minihan et al., 2004) ou até mesmo assintomáticos (Hunt e Johnson, 2007). O diagnóstico baseia-se no histórico (trauma), em sinais clínicos e em exames de 
imagem, como radiografia simples e ultrassom de abdômen e tórax. Em alguns casos, radiografia contrastada (Minihan et al., 2004) e a videolaparoscopia (Beck et al., 2004) podem ser necessárias para confirmar o diagnóstico.

Em razão de a alteração presente nas rupturas diafragmáticas ser de natureza anatômica, o tratamento cirúrgico é o único indicado (Hage e Iwasaki, 2001). As abordagens cirúrgicas mais utilizadas na medicina de pequenos animais são a laparotomia pela linha média e a toracotomia intercostal, ou a associação desses dois acessos convencionais (Hunt e Johnson, 2007). Contudo, diferentes trabalhos demonstraram a viabilidade da redução dessas hérnias via toracoscopia e laparoscopia (Beck et al., 2004; Brun et al., 2010). Ambos os acessos videocirúrgicos são seguros e efetivos, entretanto a laparoscopia permite maior facilidade na confecção da sutura intracorpórea, enquanto a toracoscopia possibilita a reposição do conteúdo de forma mais fácil (Beck et al., 2004). Diante disso, o presente relato objetivou descrever a utilização da videolaparoscopia como ferramenta auxiliar no manejo de um gato com hérnia diafragmática crônica.

\section{RELATO DE CASO}

Foi atendido, no Hospital Veterinário Universitário da Universidade Federal de Santa Maria, um gato, sem raça definida, com $3,6 \mathrm{~kg}$ e dois anos de idade, não castrado, com histórico de dificuldade respiratória e emagrecimento progressivo havia aproximadamente 30 dias. No exame físico, constatou-se desidratação leve, retração de abdômen, dispneia inspiratória moderada e abafamento dos sons cardiopulmonares à auscultação.

Os exames complementares realizados incluíram: hemograma (Ht 39,3\% [37-55]); hemoglobina (13,7g dL ${ }^{-1}$ [12-18]); VCM (39,5fL [60-77]), CHCM (34,8\% [32-36]); leucócitos totais $\left(27.300 \mu \mathrm{L}^{-1} \quad[6000-17000]\right) ; \quad$ plaquetas $\left(390.000 \mu \mathrm{L}^{-1}\right.$ [200.000-500.000]) e bioquímica sérica (creatinina: 2,2mg dL $\mathrm{mg}^{-1}[0,8-1,8]$; ureia: 58,1 $1 \mathrm{mg} \mathrm{dL}^{-1}$ [42-64]; ALT: 323UI L ${ }^{-1}$ [6-83]; FA: $58,1 \mathrm{UI} \mathrm{L}^{-1}$ [25-93]; proteínas totais: $9 \mathrm{~g} \mathrm{dL}^{-1}$ [6,0-8,0]; albumina: $2,12 \mathrm{~g} \mathrm{dL}^{-1}$ [2,1-3,3]). Após estabilização do paciente com oxigenoterapia e fluidoterapia, realizou-se exame radiográfico de tórax e abdômen, no qual foi evidenciado perda de definição da linha diafragmática e presença de vísceras abdominais (intestino e estômago) na cavidade torácica, sendo compatível com hérnia diafragmática (Fig. 1). Diante dos achados clínicos e radiológicos, optou-se pela herniorrafia diafragmática, iniciando-se a abordagem por laparoscopia.

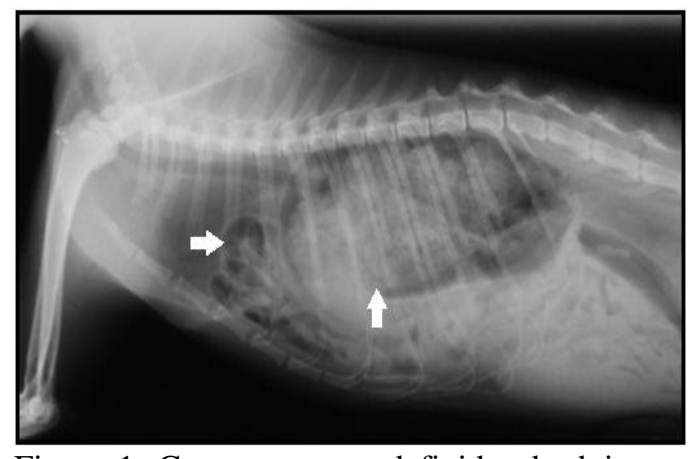

Figura 1. Gato, sem raça definida, de dois anos de idade, com hérnia diafragmática. Exame radiográfico em projeção lateral evidenciando a presença de vísceras abdominais (intestino e estômago [setas]) na cavidade torácica e perda na definição da linha diafragmática.

Como medicação pré-anestésica, foi utilizada a associação de acepromazina $0,2 \%\left(0,05 \mathrm{mg} \cdot \mathrm{kg}^{-1}\right.$, pela via intramuscular [IM]) e tramadol $\left(3 \mathrm{mg} . \mathrm{kg}^{-}\right.$ 1, IM). Para indução, utilizou-se propofol (3mg. $\mathrm{kg}^{-1}$, pela via intravenosa [IV]), seguido da intubação orotraqueal. $\mathrm{Na}$ manutenção, foi empregado isoflurano a 1CAM, vaporizado em oxigênio a $100 \%$, em circuito semiaberto.

O animal foi colocado em decúbito dorsal e, após a antissepsia do campo operatório, realizou-se a introdução do primeiro trocarte $(10 \mathrm{~mm})$ por meio de uma incisão na linha média ventral pósumbilical (distância média entre o púbis e a cicatriz umbilical), pela qual a cavidade foi insuflada com $\mathrm{CO}_{2}$ medicinal, permitindo a obtenção do pneumoperitônio (10mmHg). Posteriormente, para a utilização dos instrumentos videocirúrgicos, foi introduzido o segundo trocarte $(10 \mathrm{~mm})$ na parede abdominal lateral direita e o terceiro trocarte $(5 \mathrm{~mm})$ no abdômen esquerdo. Em seguida, inspecionou-se a cavidade abdominal com o auxílio de um endoscópio rígido $\left(10 \mathrm{~mm}\right.$ e $\left.0^{0}\right)$ e constatou-se a presença de grande defeito diafragmático. Por meio da lesão, foram observadas como estruturas herniadas: estômago (Fig. 2A), baço (Fig. 2B), considerável extensão de intestino delgado 
(Fig. 2C), omento, cólon e ceco e lobos hepático lateral esquerdo e caudado (Fig. 2D). Com o auxílio das pinças Kelly e Babcock, os tecidos e órgãos herniados foram reposicionados na cavidade abdominal.

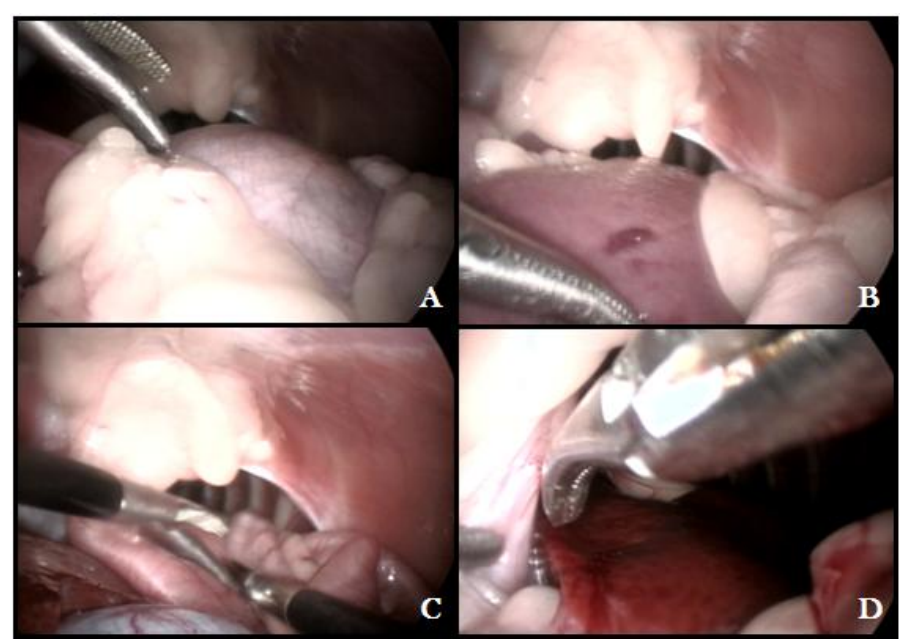

Figura 2. Gato, sem raça definida, de dois anos de idade, com hérnia diafragmática. Imagem laparoscópica durante o reposicionamento dos órgãos herniados: (A) estômago, (B) baço, (C) alças intestinais e (D) lobos hepáticos.

Após exploração laparoscópica da cavidade torácica comprovando a ausência de vísceras abdominais (Fig. 3A), tentou-se iniciar a herniorrafia com a aplicação de sutura intracorpórea em padrão isolado simples, com polipropileno 2-0, abrangendo o ponto médio do músculo diafragma de ambos os lados do defeito (Fig. 3B).
As vísceras abdominais naturalmente direcionavam-se ao tórax, dificultando a sutura intracorpórea. O animal foi, então, posicionado em cefalodeclive, no intuito de facilitar o procedimento, porém, sem sucesso. Associado a isso, não foi possível aproximar adequadamente as bordas do defeito com a sutura devido à grande tensão existente.

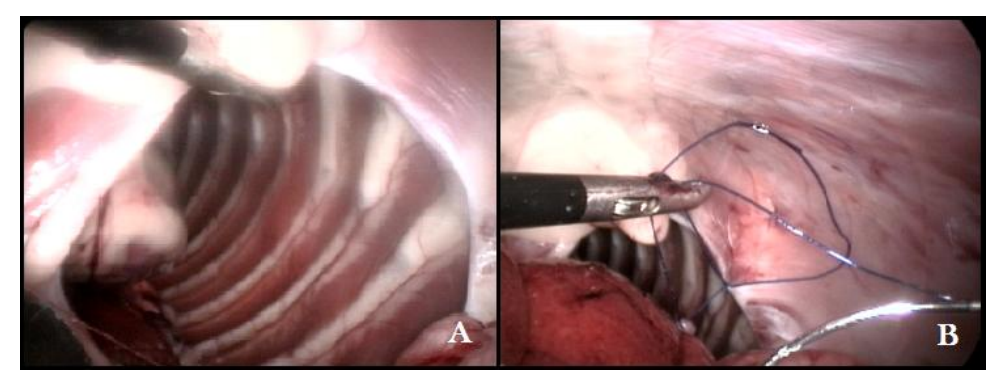

Figura 3. Gato, sem raça definida, de dois anos de idade, com hérnia diafragmática. (A) imagem laparoscópica de cavidade torácica após redução do conteúdo herniário. (B) tentativa de aplicação de sutura diafragmática intracorpórea em padrão isolado simples, com polipropileno 2-0, abrangendo o ponto médio do músculo diafragma de ambos os lados do defeito. A referida sutura foi ineficaz para aproximar as bordas do defeito diafragmático.

Diante da ineficácia da aplicação da sutura intracorpórea, optou-se por converter o procedimento para uma miniceliotomia. Para tanto, realizou-se uma incisão paracostal esquerda de aproximadamente $5 \mathrm{~cm}$, direcionada à região do defeito, procedendo-se à herniorrafia pela técnica convencional com polipropileno 2-0 em padrão colchoeiro horizontal interrompido e colchoeiro em cruz. Retirou-se o ponto anterior aplicado por laparoscopia. 
Foi aplicado dreno torácico (14Fr) no hemitórax esquerdo para drenagem pós- operatória, fixado à pele com sutura em manga chinesa. Durante a oclusão do último ponto diafragmático, procedeu-se à distensão pulmonar para reduzir o pneumotórax obtido pela insuflação. Ao término da herniorrafia, verificou-se ausência de hemorragia. Realizou-se a síntese muscular da ferida paracostal, bem como das feridas dos portais de acesso, com poliglactina 910 2-0 em padrão colchoeiro em cruz. O tecido subcutâneo e a pele foram posicionados com náilon 4-0 em padrão colchoeiro horizontal interrompido.

Não ocorreram complicações trans ou pósoperatórias associadas ao procedimento. Após a recuperação anestésica, o animal recebeu dimeticona (1 gota. $\mathrm{kg}^{-1}$, via oral [VO]), cefalotina (30mg. $\mathrm{kg}^{-1}$, pela via subcutânea) e tramadol (4mg. $\left.\mathrm{kg}^{-1}, \mathrm{VO}\right)$. Um dia após a cirurgia, foi feito exame radiográfico de tórax e abdômem, não sendo mais observadas estruturas na cavidade torácica (Fig. 4). O dreno de tórax foi removido em aproximadamente $24 \mathrm{~h}$. $\mathrm{O}$ animal recebeu alta hospitalar após 48 horas do procedimento cirúrgico. Ao final de 10 dias, encontrava-se clinicamente estável, com boa cicatrização das feridas de acesso. Aos 45 dias de pós-cirúrgico, apresentava bom estado clínico, sem alterações respiratórias e, na radiografia de tórax, não apresentava evidências de recidiva.

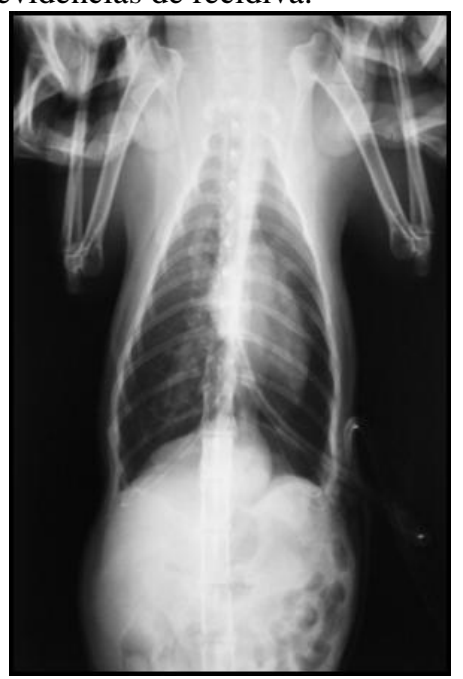

Figura 4. Gato, sem raça definida, com dois anos de idade, após herniorrafia diafragmática videoassistida. Exame radiográfico de tórax e abdômen realizado 24 horas após herniorrafia, não sendo mais observadas estruturas herniadas na cavidade torácica.

\section{DISCUSSÃO}

A causa mais comum de hérnia diafragmática em gatos é a traumática devido a acidentes automobilísticos (Hage e Iwasaki, 2001; Schmiedt et al., 2003; Minihan et al., 2004; Gibson et al., 2005; Besalti et al., 2011). No presente relato, não foi possível concluir a causa da hérnia, no entanto, em razão de o animal ter acesso livre à rua, suspeitou-se de origem traumática. Assim como o paciente deste relato, outros estudos (Schmiedt et al., 2003; Minihan et al., 2004; Gibson et al., 2005; Besalti et al., 2011) relataram machos como os mais frequentemente acometidos por traumas dessa natureza. Esses mesmos autores observaram também que o fígado foi o órgão mais encontrado na cavidade torácica de gatos com hérnia diafragmática, seguido do intestino delgado, do estômago, do omento, do baço, do pâncreas, do intestino grosso e dos rins. No presente caso, entre os órgãos citados, apenas os rins não se encontravam na cavidade torácica. Devido às características macroscópicas do defeito, verificou-se se tratar de hérnia crônica.

O exame radiográfico é o método diagnóstico mais importante para detectar hérnia diafragmática em cães e gatos, embora nem sempre seja fácil a identificação das alterações, principalmente em casos de efusão pleural concomitante (Hage e Iwasaki, 2001). No presente caso, a suspeita clínica foi confirmada pelo exame radiográfico, porém a identificação das estruturas herniadas e o tamanho da ruptura do diafragma foram possíveis apenas pela laparoscopia.

O diagnóstico de hérnia diafragmática pode ser desafiador devido ao fato de os proprietários, muitas vezes, não estarem cientes de um evento traumático, bem como em razão da pouca especificidade e sensibilidade das características clínicas e alterações na ultrassonografia e na radiografia (Minihan et al., 2004; Hunt e Johnson, 2007). No entanto, nesses casos, a laparoscopia pode ser utilizada como meio diagnóstico, uma vez que proporciona ao cirurgião um campo detalhado por meio de ampliação da imagem, permitindo a localização e, eventualmente, a correção de defeitos da musculatura (Minihan et al., 2004). A videolaparoscopia no presente caso permitiu definir o local da hérnia e promover a redução 
dos órgãos envolvidos, com o mínimo trauma operatório, o que também poderia ser feito pela abordagem convencional por cirurgia, contudo o efeito da "perda da capacitância" da cavidade abdominal poderia ser um fator de dificuldade para manutenção do conteúdo abdominal sem a distensão prévia obtida pelo pneumoperitônio. Além disso, há de se considerar que, neste caso, uma laparotomia ocasionaria maior lesão que a miniceliotomia realizada.

A cirurgia laparoscópica permite ampla exploração de ambas as cavidades através do defeito diafragmático, com mínima lesão de acesso, sendo uma vantagem importante desse acesso sobre a laparotomia mediana, a qual foi a abordagem mais utilizada para casos de hérnias diafragmáticas em cães e gatos (Schmiedt et al., 2003; Minihan et al., 2004; Gibson et al., 2005; Hunt e Johnson, 2007; Besalti et al., 2011). Entretanto, a abordagem convencional apresenta desvantagens, como: dificuldade de reposição dos órgãos para cavidade abdominal, não permissão de visibilização da cavidade torácica e a dificuldade (ou até impossibilidade) para remover das aderências entre vísceras abdominais e torácicas. Por isso, em alguns casos, torna-se necessária a extensão da laparotomia até uma esternotomia mediana (Minihan, 2004). Esse procedimento ocasiona não apenas acréscimo nas dificuldades relacionadas ao ato cirúrgico, como também maior tempo de recuperação pós-operatória dos pacientes (Hunt e Johnson, 2007). No gato deste relato, a recuperação pós-operatória foi considerada satisfatória, visto que, após 48 horas do procedimento cirúrgico, o animal não apresentava mais sinais clínicos de dispneia e de dor.

Todo procedimento cirúrgico durou 111 minutos. Esse tempo cirúrgico foi maior se comparado com o resultado obtido por Brun et al. (2010) na correção de hérnia diafragmática crônica adquirida, em uma cadela, por videolaparoscopia. Foi superior também ao estudo de Minihan et al. (2004), em que a média de tempo cirúrgico foi de 87 minutos para correção de hérnia diafragmática crônica em cães e gatos por cirurgia convencional. O tempo longo no presente caso pode ser explicado pela dificuldade da redução da hérnia, devido à grande quantidade de vísceras na cavidade torácica, e pela dificuldade da realização da sutura intracorpórea, sendo necessário acesso paracostal convencional esquerdo para auxiliar a herniorrafia.

Apesar de alguns autores recomendarem o uso de malha sintética ou retalho muscular para o fechamento da hérnia (Moreno-Egea et al., 2007), assim como no relato de Brun et al. (2010), no presente caso não foi necessário tal recurso, porque as suturas foram suficientes para uma boa aposição das margens, sem os riscos associados à manutenção de implante prostético.

Sabe-se que a cirurgia laparoscópica, em comparação com a operação convencional, reduz a produção de feridas cirúrgicas, diminui a dor pós-operatória e o desconforto, reduz o tempo de internação hospitalar e, consequentemente, reduz os custos de hospitalização (Remedios e Fergusson, 1996). No paciente deste relato, no momento em que se verificou a hérnia diafragmática, optou-se pela herniorrafia pelo acesso laparoscópico, uma vez que a videocirurgia é associada à melhor e mais rápida recuperação pós-operatória, assim como à menor produção de estímulo doloroso, à magnificação de imagens e à possibilidade de exploração ampla da cavidade peritoneal (Remedios e Fergunson, 1996). Relatos anteriores de sucesso, demonstrando que essa técnica é factível para cães (Beck et al., 2004; Brun et al., 2010), apoiaram a tentativa dos autores para a herniorrafia numa abordagem menos invasiva.

A laparoscopia permite a exploração ampla da cavidade peritoneal e a observação de todo o diafragma por meio de um único acesso (Beck et al., 2004). Na exploração diagnóstica de animais com suspeita de ruptura diafragmática, essa melhor visibilização pode ser considerada importante na escolha da técnica videocirúrgica a ser utilizada, já que a ruptura pode afetar igualmente ambos os lados do diafragma e nem sempre o local da ruptura pode ser identificado no exame radiográfico (Hage e Iwasaki, 2001; Minihan et al., 2004). No presente caso, após a visibilização da extensão da ruptura diafragmática, optou-se pela utilização da técnica de três portais de acesso, já que se tornou necessária a identificação e a manipulação visceral para reposicioná-la à posição ortotópica. Mesmo não sendo possível completar a cirurgia pelo acesso laparoscópico, tal abordagem permitiu o direcionamento do acesso para a 
realização de diminuta incisão paracostal, além da exploração do hemitórax e da possível redução do efeito da "perda de capacitância", advogando quanto à adequada opção de contar com a videocirurgia como ferramenta adjunta na resolução do caso.

Complicações no pós-operatório são comuns em herniorrafias, no entanto geralmente são transitórias e de fácil manejo, sendo o pneumotórax, que causa taquipneia e dispneia, a mais observada (Minihan et al., 2004; Schmiedt et al., 2003). Outras alterações incluem: hipotermia, vômito, anemia e hipertermia (Minihan et al., 2004). No paciente em questão, o dreno torácico foi retirado após aproximadamente 24 horas, visto que não foi observado pneumotórax no pós-operatório nem outras complicações.

A herniorrafia laparoscópica é uma técnica que vem sendo utilizada com frequência na medicina há mais de uma década (Wadhwa et al., 2005), sendo uma alternativa viável em relação aos métodos convencionais. Sua natureza minimamente invasiva, o curto tempo de hospitalização e a recuperação dos animais fazem essa modalidade diagnóstica e terapêutica se tornar interessante cada vez mais, não só para os médicos veterinários como também para os proprietários dos animais (Matyjasik et al., 2011).

Schmiedt et al. (2003) relataram que a taxa de mortalidade trans e pós-operatória não foi associada ao conteúdo da hérnia e foi maior em gatos que tiveram lesões concomitantes em outros locais. No entanto, em outros estudos (Besalti et al., 2011; Gibson et al., 2005), a taxa de mortalidade não foi diferente nos gatos com ou sem lesões simultâneas e pode estar relacionada com o local da ruptura do diafragma, a quantidade de órgãos na cavidade torácica (Besalti et al., 2011) e o tempo do trauma até a cirurgia (Gibson et al., 2005). O paciente em questão, após 45 dias do procedimento cirúrgico, apresentava bom estado clínico e não havia sinais da doença.

Conclui-se, assim, que a videolaparoscopia mostrou-se uma eficaz ferramenta diagnóstica e um recurso auxiliar no tratamento de hérnia diafragmática crônica de grande dimensão em gato.

\section{REFERÊNCIAS}

BECK, C.A.C.; PIPPI, N.L.; BRUN, M.V. et al. Laparoscopia nas hérnias diafragmáticas: estudo experimental em cães. Ciênc. Rural, v.34, p.18491855,2004

BESALTI, O.; ZEYNEP, P.; MURAT, C. et al. A retrospective study on traumatic diaphragmatic hernias in cats. Ankara Üniv. Vet. Fak. Derg., v.58, p.175-179, 2011.

BRUN, M.V.; OLIVEIRA, M.T.; OLIVEIRA, R.P. et al. Videolaparoscopia no tratamento de hérnia diafragmática crônica adquirida em uma cadela relato de caso. J. Bras. Cienc. Anim., v.3, p.13-15, 2010.

GIBSON, T.W.; BRISSON, B.A.; SEARS, W. Perioperative survival rates after surgery for diaphragmatic hernia in dogs and cats: 92 cases (19902002). J. Am. Vet. Med. Assoc., v.227, p.105-109, 2005

HAGE, M.C.F.N.S.; IWASAKI, M. Contribuição ao estudo radiográfico das rupturas diafragmáticas em cães e gatos. Clin. Vet., v.6, p.36-50, 2001.

HUNT, G.B.; JOHNSON, K.A. Hérnia diafragmática, pericárdica e hiatal. In: SLATTER, D. Manual de cirurgia de pequenos animais. 3.ed. São Paulo: Manole, p.470-487, 2007.

MATYJASIK, H.; ADAMIAK, Z.; PESTA, W. et al. Laparoscopic procedures in dogs and cats. Pol. J. Vet. Sci., v.14, p.305-316, 2011.

MINIHAN, A.C.; BERG, J.; EVANS, K.L. Chronic diaphragmatic hernia in 34 dogs and 16 cats. J. Am. Vet. Med. Assoc., v.40, p.51-63, 2004.

MORENO-EGEA, A.; GIRELA, E.; PARLORIO, E. et al. Controvérsias enel manejo actual de lãs hérnias traumáticas de pared abdominal. Cir. Espanhola, v.82, p.260-267, 2007.

REMEDIOS, A.M.; FERGUNSON, J. Minimally invasive surgery: laparoscopy and thoracoscopy in small animals. Compend. Contin. Educ. Vet., v.18, p.1191-1199, 1996.

SCHMIEDT, C.W.; KAREN, M.T.; STEVENSON, M. Traumatic diaphragmatic hernia in cats: 34 cases (1991-2001). J. Am. Vet. Med. Assoc.,v.222, p.12371240, 2003.

WADHWA, A.; SURENDRA, J.B.; SHARMA, A. $e t$ al. Laparoscopic repair of diaphragmatic hernias: experience of six cases. Asian J. Surg., v.28, p.145150, 2005. 